\title{
Do We Still Need Pioglitazone for the Management of Type 2 Diabetes Mellitus? A Peep into Risk of Bladder Cancer in Diabetic Population
}

\author{
Azharuddin M1, Tarique Faheem², Mohammad Adil' ${ }^{2}$ Pinaki Ghosh ${ }^{3}$, Manju Sharma ${ }^{2 *}$ \\ ${ }^{1}$ Department of Pharmaceutical Medicine, School of Pharmaceutical Education and Research, Jamia Hamdard, New Delhi, 110062, India \\ ${ }^{2}$ Department of Pharmacology, School of Pharmaceutical Education and Research, Jamia Hamdard, New Delhi, 110062, India
}

${ }^{3}$ Department of Pharmacology, Poona College of Pharmacy, Bharati Vidyapeeth, Pune,411038, India

Received: March 07, 2018; Accepted: March 31, 2018; Published: April 21, 2018

*Corresponding author: Manju Sharma, Associate Professor, Department of Pharmacology School of Pharmaceutical Education and Research, Jamia Hamdard, New Delhi-110062, India. Tel: +91-9810786730;E-mail: msharma@jamiahamdard.ac.in

\begin{abstract}
Pioglitazone use and risk of bladder cancer in diabetic patient is one of the major causes of morbidity and mortality. Increasing evidence from the clinical studies suggested increased risk of bladder cancer with higher dose and longer duration of pioglitazone use. In contrast, few studies reported non-significant association with pioglitazone use and risk of bladder cancer in T2DM patients. Therefore, we aimed to review and summarize the available clinical data describing the risk of bladder cancer associated with pioglitazone treatment.
\end{abstract}

Keywords: Type 2 diabetes mellitus; pioglitazone; bladder cancer

\section{Review}

Pioglitazone belongs to thiazolidinedione class and acts as insulin sensitizer, widely used for the management of type 2 diabetes mellitus (T2DM). Pioglitazone, targets peroxisome proliferators-activated receptor-gamma (PPAR- $\gamma$ ) protein, a key transcription factor for adipogenesis and glucose homeostasis [1]. Despite the beneficial effect of pioglitazone use in glycemic control, its extended use has been allied with various adverse events and questioned owing to safety concerns. Bladder cancer is one of the risk factors associated with the pioglitazone use [2]. According to American Cancer Society, 79,030new cases causing about 16,870 deaths were reported from bladder cancer in the United State (US) for 2017. Bladder cancer is the fourth most common cancer in men and less common in women, over all accounting for approximately $5 \%$ of all new cancers in the USA [3]. Both diabetes and bladder cancer are the major causes of morbidity and mortality, resulting in substantial health economic burden. PRO active study was the first study, which reported the incidence of bladder cancer, where 14 cases of bladder cancer were observed in the pioglitazone treatment versus 5 cases of placebo arms [4]. An interim report from cohort study reviewed by Food and Drug Administration (FDA) revealed that the risk of bladder cancer was not associated with short-term use of pioglitazone, whereas more than 2 years use of pioglitazone associated with increased risk of bladder cancer in diabetic patients [5]. As a result, in 2011, US FDA updated the public safety report regarding the pioglitazone use for more than 1 year with increased risk of bladder cancer. Additionally, recommendations were given to healthcare professionals against prescribing pioglitazone in patients with active bladder cancer and prescribe it with caution in patients having history of bladder cancer [6]. In line with US FDA stand, after a finding from cohort study conducted in France, European Medicines Agency (EMA) also decided to suspend use of pioglitazone in diabetic patients [7].

The ongoing results from the several studies suggested that pioglitazone use was associated with increased risk of bladder cancer $[8,9]$. On the other hand, few studies reported no risk of bladder cancer with pioglitazone use [10,11]. For instance, a recent population based cohort study conducted in United Kingdom (UK) generated 689,616 person years of follow-up, during which 622 patients were newly diagnosed as having bladder cancer (crude incidence 90.2 per 100,000 person years). When compared with other anti-diabetic medications, pioglitazone use was associated with an increased risk of bladder cancer (121.0 versus 88.9 per 100,000 person years) with hazard ratio (HR) 1.63, 95\% confidence interval (CI) 1.22- 2.19. This study also reported that risk of bladder cancer with pioglitazone may vary in a dose and duration dependent manner [8]. In addition, an epidemiological study identified 1,161,443 prevalent and 320,090 incident diabetics with mean age $=75.1$ years, mean follow-up time $=38.0$ months, and $20.2 \%$ filled a prescription for pioglitazone. Results from this study reported a positive association between bladder cancer and duration of pioglitazone use in the prevalent cohort $(\mathrm{P}=0.008)$, with $\geq 24$ months of pioglitazone exposure increasing the incidence of bladder cancer $16 \%$ (95\% CI 0-35\%) compared to non-user [12]. Interestingly, a recent meta-analysis of 15 observational studies conducted by Adil et al. [13] which included 2,470,397 diabetic patient and 
12,138 bladder cancer cases reported risk of bladder cancer with pioglitazone use (HR $=1.20 ; 95 \%$ CI 1.09-1.31; $\mathrm{P}<0.0001)$. Subgroup analysis of this meta-analysis also reported that dose $(10,501-28,000 \mathrm{mg}$ and $>28,000 \mathrm{mg}$ ) (HR $=1.27$; $95 \%$ CI $1.05-$ 1.54; $\mathrm{P}<0.01$ and $\mathrm{HR}=1.68 ; 95 \%$ CI 1.36-2.08; $\mathrm{P}<0.00001$; respectively) and duration (12-24 and $>24$ months) (HR $=1.43$; 95\% CI 1.19-1.71; $\mathrm{P}=0.0001$ and $\mathrm{HR}=1.58 ; 95 \%$ CI $1.27-1.97$ $\mathrm{P}<0.0001$ respectively) of pioglitazone use is associated with risk of bladder cancer. On the contrary, a retrospective cohort study performed by Korhonen et al [14]. uses datasets from four European countries evaluating association between pioglitazone use and bladder cancer risk in T2DM patients. Results from this cohort study found non-significant association between bladder cancer risk and pioglitazone use when compared to nonusers (HR $=0.99$; 95\% CI 0.75-1.30) and (HR $=1.00$, 95\% CI 0.831.21 ) in the nearest and multiple match cohorts, respectively. Similarly, increase in cumulative dose ( $>40000 \mathrm{mg}$ ) and duration ( $>48$ months) of pioglitazone exposure was also not associated with risk of bladder cancer $(\mathrm{HR}=0.65 ; 95 \%$ CI $0.33-1.26$ and $\mathrm{HR}=0.86 ; 95 \%$ CI $0.44-1.66$,respectively) in the nearest match cohort.

Another, recent meta-analysis conducted by Filipova and colleague reported no link of bladder cancer risk with pioglitazone use, Relative risk; RR $=1.13$; 95\% CI 0.96-1.33 and HR $=1.07$; 95\% CI 0.96-1.18), and suggested hypothetically increased risk of bladder malignancy should be attributed to other factors [11].

Evidence surrounding the association between pioglitazone use and risk of bladder cancer requires cautious interpretation because of inconsistent results. The evidence from real world data turned out to be insufficient to prove association between pioglitazone use and risk of bladder cancer. So far, no randomized controlled trials (RCTs) have been performed to evaluate the impact of pioglitazone on bladder cancer in diabetic patients which makes it difficult to draw conclusions on whether the association between bladder cancer risk and pioglitazone is related to pioglitazone treatment itself or to the patient's disease state. However, patients with uncontrolled diabetes may need to use pioglitazone because of scarcity of PPAR- $\gamma$ agonist. Such knowledge would be important for trade-offs and decision making when this treatment is being considered. Therefore, welldesigned RCTs with long-term follow up are required to explore the effect of pioglitazone on bladder cancer risk.

\section{References}

1. Rizos CV, Kei A, Elisaf MS. The current role of thiazolidinediones in diabetes management. Arch Toxicol. 2016;90:1861-1881. Doi: 10.1007/s00204- 016-1737-4

2. Lewis JD, Habel LA, Queensberry CP, Strom BL, Peng T, Hedderson MM, et al. Pioglitazone use and risk of bladder cancer and other common cancers in persons with diabetes. JAMA. 2015;314(3):265-277. Doi: 10.1001/jama.2015.7996
3. American cancer society. Last medical review: January 26, 2016. Last revised: May 1, 2017.

4. Dormandy JA, Charbonnel B, Eckland DJ, Erdmann E, Massi-Benedetti $\mathrm{M}$, Moules IK, et al. Secondary prevention of macrovascular events in patients with type 2 diabetes in the PROactive Study (PRO spective pioglit Azone Clinical Trial in macroVascular Events):a randomised controlled trial[J]. Lancet. 2005;366(9493):1279-1289. Doi: 10.1016/ S0140- 6736(05)675289

5. Lewis JD, Ferrara A, Peng T, Hedderson M, Bilker WB, Quesenberry CP Jr, et al. Risk of bladder cancer among diabetic patients treated with pioglitazone: interim report of a longitudinal cohort study[J]. Diabetes Care. 2011;34(4):916-922. Doi: 10.2337/dc10-1068

6. Food and Drug Administration. FDA drug safety communication: updated drug labels for pioglitazone-containing medicines. 2017.

7. European Medicines Agency. Questions and answers on the review of pioglitazone containing medicines (Actos, Glustin, Competact, Glubrava and Tandemact). 2011.

8. Tuccori M, Filion KB, Yin H, et al. Pioglitazone use and risk of bladder cancer: population based cohort study[J]. BMJ, 2016;352: i1541. Doi: 10.1136/bmj. i1541

9. Neumann A, Weill A, Ricordeau P, et al. Pioglitazone and risk of bladder cancer among diabetic patients in France: a population-based cohort study[J]. Diabetologia. 2012;55(7):1953-1962. Doi: 10.1007/s00125012-2538-9

10. Gupta S, Gupta K, Ravi R Fagot JP, Alla F, Allemand H. Pioglitazone and the risk of bladder cancer: An Indian retrospective cohort study[J]. Indian J Endocrinol Metab. 2015;19:639-643. Doi: 10.4103/22308210.163187

11. Elena Filipova, Katya Uzunova, Krassimir Kalinov, Toni Vekov. Pioglitazone and the Risk of Bladder Cancer: A Meta-Analysis[J]. Diabetes Ther, 2017;8(4):705-726. Doi: 10.1007/s13300-017-0273-4

12. Mackenzie TA, Zaha R, Smith J, Karagas MR, Morden NE. Diabetes pharmacotherapies and bladder cancer: a Medicare epidemiologic study[J]. Diabetes Therapy. 2016;7(1):61-73. Doi: 10.1007/s13300016-0152-4

13. Mohammad Adil, Rashid Ali Khan, Pinaki Ghosh, Shiva Kumar Venkata, Amit Dattatraya Kandhare, Manju Sharma. Pioglitazone and risk of bladder cancer in type 2 diabetes mellitus patients: A systematic literature review and meta-analysis of observational studies using real-world data[J]. Clinical Epidemiology and Global Health. 2017. Doi: 10.1016/j.cegh.2017.08.002

14. Korhonen P, Heintjes EM, Williams R, et al. Pioglitazone use and risk of bladder cancer in patients with type 2 diabetes: retrospective cohort study using datasets from four European countries[J]. BMJ. 2016;16:354:i3903 Doi: 10.1136/bmj.i3903 